\title{
HYPERBOLIC HYPERSURFACES IN THE COMPLEX PROJECTIVE SPACES OF LOW DIMENSIONS
}

\author{
MANABU SHIROSAKI
}

\section{§1. Introduction}

There have been a number of results for hyperbolic hypersurfaces in the complex projective spaces (cf. $[\mathrm{AS}],[\mathrm{BG}],[\mathrm{D}],[\mathrm{K}],[\mathrm{MN}],[\mathrm{N}],[\mathrm{S}]$ and $[\mathrm{Z}]$ ). In particular, J. P. Demailly [D] constructed a remarkable example of hyperbolic hypersurfaces of degree 11 in $\boldsymbol{P}^{3}(\boldsymbol{C})$. On the other hand, the author [S] gave hyperbolic hypersurfaces of degree $13^{n}$ in $\boldsymbol{P}^{n}(\boldsymbol{C})$ whose complements are complete hyperbolic and hyperbolically imbedded in $\boldsymbol{P}^{n}(\boldsymbol{C})$. In this paper, we give hyperbolic hypersurfaces in the complex projective spaces of dimension 2,3 and 4. For example, we construct hyperbolic hypersurfaces in $\boldsymbol{P}^{3}(\boldsymbol{C})$ of degree 31 whose complements are complete hyprebolic and hyperbolically imbedded in $\boldsymbol{P}^{3}(\boldsymbol{C})$, and hyperbolic hypersurface of degree 36 in $\boldsymbol{P}^{4}(\boldsymbol{C})$.

Acknowledgment. The author would like to thank the referee for many helpful comments.

\section{§2. A holomorphic mapping into a hypersurface in $\boldsymbol{P}^{n}(\boldsymbol{C})$}

Let $n, q$ and $d$ be positive integers such that $q \geq n+1$ and $d \geq(q-1)^{2}$. Let $V$ be a set of $q$ column vectors in $C^{n+1}$. We make the following assumptions.

(A1) The vectors in $V$ are in general position.

(A2) Take any $k$ with $0 \leq k \leq \min \{n, q-n-2\}$. Then, for any distinct vectors $\boldsymbol{v}_{0}, \ldots, \boldsymbol{v}_{n}, \boldsymbol{u}_{0}, \ldots, \boldsymbol{u}_{k}$ in $V$ and any $d$-th roots of $\omega_{0}, \ldots, \omega_{k}$ of -1 , the $n+1$ vectors $\boldsymbol{v}_{j}-\omega_{j} \boldsymbol{u}_{j}(0 \leq j \leq k)$ and $\boldsymbol{v}_{j}(k+1 \leq j \leq n)$ are linearly independent.

(A3) Take any $k$ with $1 \leq k \leq \min \{n, q-n-1\}$. Then, for any distinct vectors $\boldsymbol{v}_{0}, \ldots, \boldsymbol{v}_{n}, \boldsymbol{u}_{1}, \ldots, \boldsymbol{u}_{k}$ in $V$

$$
\sum_{j=1}^{k}\left\{\frac{\operatorname{det}\left(\boldsymbol{u}_{j}, \boldsymbol{v}_{1}, \ldots, \boldsymbol{v}_{n}\right)}{\operatorname{det}\left(\boldsymbol{v}_{0}, \boldsymbol{v}_{1}, \ldots, \boldsymbol{v}_{n}\right)}\right\}^{d}+1 \neq 0 .
$$

Received April 23, 1999; revised January 28, 2000. 
We define a hypersurface $S(V)$ assoicated with $V$ in $\boldsymbol{P}^{n}(\boldsymbol{C})$ by

$$
\sum_{\boldsymbol{v} \in V}(\boldsymbol{z} \cdot \boldsymbol{v})^{d}=0
$$

where $\boldsymbol{z} \cdot \boldsymbol{v}=v_{0} z_{0}+\cdots+v_{n} z_{n}$ for $\boldsymbol{v}={ }^{t}\left(v_{0}, \ldots, v_{n}\right)$.

Now, let $f$ be a holomorphic mapping of $\boldsymbol{C}$ into $\boldsymbol{P}^{n}(\boldsymbol{C})$ with a reduced representation $\boldsymbol{f}=\left(f_{0}, \ldots, f_{n}\right)$ such that $f(\boldsymbol{C}) \subset S(V)$, i.e.,

$$
\sum_{\boldsymbol{v} \in V}(\boldsymbol{f} \cdot \boldsymbol{v})^{d} \equiv 0
$$

where $\boldsymbol{f} \cdot \boldsymbol{v}=v_{0} f_{0}+\cdots+v_{n} f_{n}$ for $\boldsymbol{v}={ }^{t}\left(v_{0}, \ldots, v_{n}\right)$.

The following generalized Borel's lemma is due to H. Fujimoto and M. Green (cf. [F, Corollary 6.4] and [G, p. 70]):

LEMMA 2.1. Let $f: C \rightarrow S(V)$ be a holomorphic mapping. If $d \geq(q-1)^{2}$, then there exists a decomposition $V=V_{0} \cup V_{1} \cup \cdots \cup V_{\ell}$ of $V$ with $\sharp V \geq 2(1 \leq j \leq \ell)$ such that

(i) $\boldsymbol{v} \in V_{0}$ if and only if $\boldsymbol{f} \cdot \boldsymbol{v} \equiv 0$,

(ii) for $\boldsymbol{u}, \boldsymbol{v} \in V \backslash V_{0},(\boldsymbol{f} \cdot \boldsymbol{u}) /(\boldsymbol{f} \cdot \boldsymbol{v})$ is constant if and only if there exists $j$ with $1 \leq j \leq \ell$ such that $\boldsymbol{u}, \boldsymbol{v} \in V_{j}$,

(iii) $\sum_{v \in V_{j}}(\boldsymbol{f} \cdot \boldsymbol{v})^{d} \equiv 0$ for each $j$ with $1 \leq j \leq \ell$.

Put $k_{J}:=\sharp V_{J}-1$ for $1 \leq j \leq \ell$ and $k_{0}=\sharp V_{0}$. Then, we may assume $k_{1} \geq \cdots \geq k_{\ell}$ by changing indices. In this situation, we call $V=V_{0} \cup V_{1} \cup \cdots$ $\cup V_{\ell}$ the first kind of decomposition of $V$ by $f$, and $\left(k_{0} ; k_{1}+1, \ldots, k_{\ell}+1\right)$ its type. Set

$$
V_{0}=\left\{\boldsymbol{v}_{1}^{(0)}, \ldots, \boldsymbol{v}_{k_{0}}^{(0)}\right\}
$$

and

$$
V_{J}=\left\{\boldsymbol{v}_{0}^{(j)}, \ldots, \boldsymbol{v}_{k_{j}}^{(j)}\right\} \quad(1 \leq j \leq \ell) .
$$

Then, by Lemma 2.1, there exist nonzero constants $\omega_{\mu}^{(j)}$ such that

$$
\boldsymbol{f} \cdot\left(\boldsymbol{v}_{\mu}^{(j)}-\omega_{\mu}^{(j)} \boldsymbol{v}_{0}^{(j)}\right) \equiv 0 \quad\left(1 \leq \mu \leq k_{J}, 1 \leq j \leq \ell\right)
$$

and

$$
1+\left(\omega_{1}^{(j)}\right)^{d}+\cdots+\left(\omega_{k_{j}}^{(j)}\right)^{d}=0 \quad(1 \leq j \leq \ell) .
$$

For brevity, we write $\boldsymbol{w}_{\mu}^{(j)}$ for $\boldsymbol{v}_{\mu}^{(j)}-\omega_{\mu}^{(j)} \boldsymbol{v}_{\mu}^{(j)}$. The equations of (2.1) and $\boldsymbol{f} \cdot \boldsymbol{v}_{\mu}^{(0)} \equiv 0\left(1 \leq \mu \leq k_{0}\right)$ can be represented as

$$
\boldsymbol{f}\left(\boldsymbol{v}_{1}^{(0)}, \ldots, \boldsymbol{v}_{k_{0}}^{(0)}, \boldsymbol{w}_{1}^{(1)}, \ldots, \boldsymbol{w}_{k_{1}}^{(1)}, \ldots, \boldsymbol{w}_{1}^{(\ell)}, \ldots, \boldsymbol{w}_{k_{\ell}}^{(\ell)}\right) \equiv(0, \ldots, 0) .
$$

LEMMA 2.2. If there exists a nonconstant holomorphic mapping $f: C \rightarrow$ $S(V)$, then 
(i) $0 \leq k_{0} \leq n-1$,

(ii) $1 \leq k_{J} \leq n-1$ for $1 \leq j \leq \ell$,

(iii) the rank of the matrix

$$
\left(\boldsymbol{v}_{1}^{(0)}, \ldots, \boldsymbol{v}_{k_{0}}^{(0)}, \boldsymbol{w}_{1}^{(1)}, \ldots, \boldsymbol{w}_{k_{1}}^{(1)}, \ldots, \boldsymbol{w}_{1}^{(\ell)}, \ldots, \boldsymbol{w}_{k_{\ell}}^{(\ell)}\right)
$$

is not greater than $n-1$.

Proof. The assertions (i) and (ii) follow from the assertion (iii) and the assumptions (A1) and (A2). Hence we prove only (iii).

We assume that the rank of the matrix $\left(\boldsymbol{v}_{1}^{(0)}, \ldots, \boldsymbol{v}_{k_{0}}^{(0)}, \boldsymbol{w}_{1}^{(1)}, \ldots, \boldsymbol{w}_{k_{1}}^{(1)}, \ldots\right.$, $\left.\boldsymbol{w}_{1}^{(\ell)}, \ldots, \boldsymbol{w}_{k_{\ell}}^{(\ell)}\right)$ is greater than $n-1$. Then there exist a non-singular $(n+1) \times$ $(n+1)$-matrix $P$ and an entire function $h$ such that $f P=(0, \ldots, 0, h)$. Hence, $\boldsymbol{f}=\left(c_{0}: \cdots: c_{n}\right)$, where $\left(c_{0}, \ldots, c_{n}\right)$ is the $(n+1)$-th row of $P^{-1}$. Q.E.D.

In the rest of this section, we assume that there exists a nonconstant holomorphic mapping $f: C \rightarrow S(V)$.

LEMMA 2.3. $k_{0}+k_{1} \leq n-2$.

Proof. Assume the contrary. Then $k_{0}+k_{1} \geq n-1$.

(i) The case of $k_{0}+k_{1} \geq n$. In this case, by Lemma 2.2, there exist not all zero constants $a_{1}, \ldots, a_{k_{1}}, b_{1} \ldots, b_{n-k_{1}}$ such that

$$
\begin{aligned}
& a_{1} \boldsymbol{w}_{1}^{(1)}+\cdots+a_{k_{1}} \boldsymbol{w}_{k_{1}}^{(1)}+b_{1} \boldsymbol{v}_{1}^{(0)}+\cdots+b_{n-k_{1}} \boldsymbol{v}_{n-k_{1}}^{(1)} \\
& \quad=a_{1}\left(\boldsymbol{v}_{1}^{(1)}-\omega_{1}^{(1)} \boldsymbol{v}_{0}^{(1)}\right)+\cdots+a_{k_{1}}\left(\boldsymbol{v}_{k_{1}}^{(1)}-\omega_{k_{1}}^{(1)} \boldsymbol{v}_{0}^{(1)}\right)+b_{1} \boldsymbol{v}_{1}^{(0)}+\cdots+b_{n-k_{1}} \boldsymbol{v}_{n-k_{1}}^{(1)}=\mathbf{0} .
\end{aligned}
$$

This contradicts (A1).

(ii) The case of $k_{0}+k_{1}=n-1$. In this case, $\ell \geq 2$. It follows from Lemma 2.2 that there exist constants $a_{j 1}, \ldots, a_{j k_{1}}, b_{j 1}, \ldots, b_{j k_{0}}$ such that

$$
\begin{aligned}
\boldsymbol{v}_{j}^{(2)}- & \omega_{j}^{(2)} \boldsymbol{v}_{0}^{(2)} \\
& =a_{j 1} \boldsymbol{w}_{1}^{(1)}+\cdots+a_{j k_{1}} \boldsymbol{w}_{k_{1}}^{(1)}+b_{j 1} \boldsymbol{v}_{1}^{(0)}+\cdots+b_{j k_{0}} \boldsymbol{v}_{k_{0}}^{(1)} \\
& =a_{j 1}\left(\boldsymbol{v}_{1}^{(1)}-\omega_{1}^{(1)} \boldsymbol{v}_{0}^{(1)}\right)+\cdots+a_{j k_{1}}\left(\boldsymbol{v}_{k_{1}}^{(1)}-\omega_{k_{1}}^{(1)} \boldsymbol{v}_{0}^{(1)}\right)+b_{j 1} \boldsymbol{v}_{1}^{(0)}+\cdots+b_{j k_{0}} \boldsymbol{v}_{k_{0}}^{(1)}
\end{aligned}
$$

for $1 \leq j \leq k_{2}$. By Cramer's formula we have

$$
\omega_{j}^{(2)}=\frac{\operatorname{det}\left(\boldsymbol{v}_{j}^{(2)}, \boldsymbol{v}_{0}^{(1)}, \ldots, \boldsymbol{v}_{k_{1}}^{(1)}, \boldsymbol{v}_{1}^{(0)}, \ldots, \boldsymbol{v}_{k_{0}}^{(0)}\right)}{\operatorname{det}\left(\boldsymbol{v}_{0}^{(2)}, \boldsymbol{v}_{0}^{(1)}, \ldots, \boldsymbol{v}_{k_{1}}^{(1)}, \boldsymbol{v}_{1}^{(0)}, \ldots, \boldsymbol{v}_{k_{0}}^{(0)}\right)},
$$

which contradicts (2.2) and (A3).

Q.E.D.

LEMMA 2.4. $k_{0}+\sharp\left\{j ; 2 \leq j \leq \ell\right.$ and $\left.k_{J}=1\right\} \leq n-2$. 
Proof. Assume $k_{0}+\sharp\left\{j ; 2 \leq j \leq \ell\right.$ and $\left.k_{j}=1\right\} \geq n-1$. Take $m$ such that $2 \leq m \leq \ell, \ell-m+1+k_{0}=n-1$ and $k_{m}=\cdots=k_{\ell}=1$. Then, by Lemma $2.2, \boldsymbol{v}_{1}^{(1)}-\omega_{1}^{(1)} \boldsymbol{v}_{0}^{(1)}, \boldsymbol{v}_{1}^{(0)}, \ldots, \boldsymbol{v}_{k_{0}}^{(0)}, \boldsymbol{v}_{1}^{(m)}-\omega_{1}^{(m)} \boldsymbol{v}_{0}^{(m)}, \ldots, \boldsymbol{v}_{1}^{(\ell)}-\omega_{1}^{(\ell)} \boldsymbol{v}_{0}^{(\ell)}$ are linearly dependent, which contradicts (A2).

Q.E.D.

LEMMA 2.5. $k_{0}+k_{1}+\sharp\left\{j ; 2 \leq j \leq \ell\right.$ and $\left.k_{J}=1\right\} \leq n-1$.

Proof. By Lemma 2.3, it is enough to consider the case where $\ell \geq 2$. Assume $k_{0}+k_{1}+\sharp\left\{j ; 2 \leq j \leq \ell\right.$ and $\left.k_{J}=1\right\} \geq n$. Take $m$ such that $2 \leq m \leq \ell, \ell-m+1+k_{0}+k_{1}=n$ and $k_{m}=\cdots=k_{\ell}=1$. Then, by Lemma $2.2, \boldsymbol{v}_{1}^{(0)}, \ldots, \boldsymbol{v}_{k_{0}}^{(0)}, \boldsymbol{v}_{1}^{(1)}-\omega_{1}^{(1)} \boldsymbol{v}_{0}^{(1)}, \ldots, \boldsymbol{v}_{k_{1}}^{(1)}-\omega_{k_{1}}^{(1)} \boldsymbol{v}_{0}^{(1)}, \boldsymbol{v}_{1}^{(m)}-\omega_{1}^{(m)} \boldsymbol{v}_{0}^{(m)}, \ldots, \boldsymbol{v}_{1}^{(\ell)}-\omega_{1}^{(\ell)} \boldsymbol{v}_{0}^{(\ell)}$ are linearly dependent. This contradicts (A2).

\section{§3. Hyperbolicity of hypersurfaces in $P^{n}(C)(n=2,3$ and 4)}

In this section, we prove the hyperbolicity of $S(V)$ in the case where $n=2,3$ and 4. By Brody's criterion for hyperbolicity ([B]), it surffices to show that there exists no nonconstant holomorphic mapping $f: C \rightarrow S(V)$.

THEOREM 3.1. If $n=2$, then $S(V)$ is hyperbolic.

Proof. Suppose that $S(V)$ is not hyperbolic. Then there exists a nonconstant holomorphic mapping $f: C \rightarrow S(V)$. By Lemma 2.3, we see $k_{0}+k_{1} \leq 0$. This contradicts $k_{1} \geq 1$.

Q.E.D.

The least degree of the hyperbolic hypersurfaces in Theorem 3.1 is 4 .

THEOREM 3.2. If $n=3$ and $q \geq 5$, then $S(V)$ is hyperbolic.

Proof. Suppose that $S(V)$ is not hyperbolic. By Lemma 2.3, we see $k_{0}+k_{1} \leq 1$. This implies that $k_{0}=0$ and $k_{1}=1$. Since $k_{1} \geq \cdots \geq k_{\ell} \geq 1$, we have that $k_{1}=\cdots=k_{\ell}=1$ and hence $q=2 \ell$. If $q \geq 5$ is odd, then no such decomposition occurs. If $q \geq 5$ is even, we get

$$
k_{0}+\sharp\left\{j ; 1 \leq j \leq \ell \text { and } k_{j}=1\right\} \geq 3,
$$

which contradicts Lemma 2.4 .

Q.E.D.

The least degree of the hyperbolic hypersurfaces in Theorem 3.2 is 16 .

THEOREM 3.3. If $n=4$ and $q=7$, then $S(V)$ is hyperbolic.

Proof. Suppose that $S(V)$ is not hyperbolic. By Lemma 2.3, we see $k_{0}+$ $k_{1} \leq 2$. Since $q=7$, it is easy to see that the only possible types of decomposition of the first type are of types $(0 ; 3,2,2)$ and $(1 ; 2,2,2)$. For these two 
types, we have

$$
k_{0}+k_{1}+\sharp\left\{j ; 2 \leq j \leq 3 \text { and } k_{\jmath}=1\right\} \geq 4 .
$$

This contradicts Lemma 2.5 .

Q.E.D.

K. Masuda and J. Noguchi $[\mathrm{MN}]$ gave an example of hyperbolic hypersurface of degree 196 in $\boldsymbol{P}^{4}(\boldsymbol{C})$. The least degree of hyperbolic hypersurfaces in Theorem 3.3 is 36 .

\section{§4. A holomorphic mapping omitting a hypersurface}

Let $n, q$ and $d$ be positive integers such that $q \geq n+1$ and $d \geq$ $(q-1) q+1$. Let $V$ be as in $\S 2$. Assume that (A1), (A2) and (A3). Let $f: \boldsymbol{C} \rightarrow \boldsymbol{P}^{n}(\boldsymbol{C})$ with a reduced representation $\boldsymbol{f}=\left(f_{0}, \ldots, f_{n}\right)$ such that $f(\boldsymbol{C}) \cap$ $S(V)=\emptyset$, i.e.,

$$
\sum_{v \in V}(\boldsymbol{f} \cdot \boldsymbol{v})^{d} \equiv \alpha^{d}
$$

for an entire function $\alpha$ without zeros. The following Lemma due to M. Green plays an essential role (cf. [G, p. 73]):

LEMMA 4.1. Let $f: \boldsymbol{C} \rightarrow \boldsymbol{P}^{n}(\boldsymbol{C}) \backslash S(V)$ be a holomorphic mapping. If $d \geq(q-1) q+1$, then there exists a decomposition $V=V_{0} \cup V_{1} \cup \cdots \cup V_{\ell} \cup V_{\ell+1}$ of $V$ with $V_{\ell+1} \neq \emptyset$ such that

(i) $\sharp V_{J} \geq 2(1 \leq j \leq \ell)$,

(ii) $\boldsymbol{v} \in V_{0}$ if and only if $\boldsymbol{f} \cdot \boldsymbol{v} \equiv 0$,

(iii) for $\boldsymbol{u}, \boldsymbol{v} \in V \backslash V_{0},(\boldsymbol{f} \cdot \boldsymbol{u}) /(\boldsymbol{f} \cdot \boldsymbol{v})$ is constant if and only if there exists $j$ with $1 \leq j \leq \ell+1$ such that $\boldsymbol{u}, \boldsymbol{v} \in V_{J}$,

(iv) $\sum_{v \in V_{l}}(\boldsymbol{f} \cdot \boldsymbol{v})^{d} \equiv 0$ for each $1 \leq j \leq \ell$,

(v) $v \in V_{\ell+1}$ if and only if $(\boldsymbol{f} \cdot \boldsymbol{v}) / \alpha$ is constant,

(vi) $\sum_{v \in V_{t+1}}(\boldsymbol{f} \cdot \boldsymbol{v})^{d}=\alpha^{d}$.

Put $k_{J}:=\sharp V_{J}-1$ for $1 \leq j \leq \ell+1$ and $k_{0}:=\sharp V_{0}$. We may assume that $k_{1} \geq \cdots \geq k_{\ell}$. We call $V=V_{0} \cup V_{1} \cup \cdots \cup V_{\ell} \cup V_{\ell+1}$ the second kind of decomposition of $V$ by $f$, and $\left(k_{0} ; k_{1}+1, \ldots, k_{\ell}+1 ; k_{\ell+1}+1\right)$ its type.

Set

$$
V_{0}=\left\{\boldsymbol{v}_{1}^{(0)}, \ldots, \boldsymbol{v}_{k_{0}}^{(0)}\right\}
$$

and

$$
V_{J}=\left\{\boldsymbol{v}_{0}^{(j)}, \ldots, \boldsymbol{v}_{k_{j}}^{(j)}\right\} \quad(1 \leq j \leq \ell+1) .
$$

Then, by Lemma 4.1 , there exist nonzero constants $\omega_{\mu}^{(j)}$ such that

$$
\boldsymbol{f} \cdot\left(\boldsymbol{v}_{\mu}^{(j)}-\omega_{\mu}^{(j)} \boldsymbol{v}_{0}^{(j)}\right) \equiv 0 \quad\left(1 \leq \mu \leq k_{J}, 1 \leq j \leq \ell\right)
$$


and

$$
1+\left(\omega_{1}^{(j)}\right)^{d}+\cdots+\left(\omega_{k_{j}}^{(j)}\right)^{d}=0 \quad(1 \leq j \leq \ell) .
$$

If $k_{\ell+1} \geq 1$, then there exist nonzero constants $\omega_{\mu}^{(\ell+1)}$ such that

$$
\boldsymbol{f} \cdot\left(\boldsymbol{v}_{\mu}^{(\ell+1)}-\omega_{\mu}^{(\ell+1)} \boldsymbol{v}_{0}^{(\ell+1)}\right) \equiv 0 \quad\left(1 \leq \mu \leq k_{\ell+1}\right)
$$

However, there is no relation corresponding to (4.2) for $\omega_{\mu}^{(\ell+1)}$. By (4.1), (4.3) and $\boldsymbol{f} \cdot \boldsymbol{v}_{\mu}^{(0)} \equiv 0$, we have the following: If $k_{\ell+1}=0$, then

$$
\boldsymbol{f}\left(\boldsymbol{v}_{1}^{(0)}, \ldots, \boldsymbol{v}_{k_{0}}^{(0)}, \boldsymbol{w}_{1}^{(1)}, \ldots, \boldsymbol{w}_{k_{1}}^{(1)}, \ldots, \boldsymbol{w}_{1}^{(\ell)}, \ldots, \boldsymbol{w}_{k_{\ell}}^{(\ell)}\right) \equiv(0, \ldots, 0),
$$

and if $k_{\ell+1} \geq 1$, then

$$
\boldsymbol{f}\left(\boldsymbol{v}_{1}^{(0)}, \ldots, \boldsymbol{v}_{k_{0}}^{(0)}, \boldsymbol{w}_{1}^{(1)}, \ldots, \boldsymbol{w}_{k_{1}}^{(1)}, \ldots, \boldsymbol{w}_{1}^{(\ell+1)}, \ldots, \boldsymbol{w}_{k_{\ell+1}}^{(\ell+1)}\right) \equiv(0, \ldots, 0) .
$$

LEMma 4.2. If there exists a nonconstant holomorphic mapping $f: C \rightarrow$ $\boldsymbol{P}^{n}(\boldsymbol{C}) \backslash S(V)$, then

(i) $0 \leq k_{0} \leq n-1$,

(ii) $1 \leq k_{J} \leq n-1(1 \leq j \leq \ell)$,

(iii) if $k_{\ell+1}=0$, the rank of the matrix

$$
\left(\boldsymbol{v}_{1}^{(0)}, \ldots, \boldsymbol{v}_{k_{0}}^{(0)}, \boldsymbol{w}_{1}^{(1)}, \ldots, \boldsymbol{w}_{k_{1}}^{(1)}, \ldots, \boldsymbol{w}_{1}^{(\ell)}, \ldots, \boldsymbol{w}_{k_{\ell}}^{(\ell)}\right)
$$

is not greater than $n-1$, and if $k_{\ell+1} \geq 1$, the rank of the matrix

$$
\left(\boldsymbol{v}_{1}^{(0)}, \ldots, \boldsymbol{v}_{k_{0}}^{(0)}, \boldsymbol{w}_{1}^{(1)}, \ldots, \boldsymbol{w}_{k_{1}}^{(1)}, \ldots, \boldsymbol{w}_{1}^{(\ell+1)}, \ldots, \boldsymbol{w}_{k_{\ell+1}}^{(\ell+1)}\right)
$$

is not greater than $n-1$,

(iv) $0 \leq k_{\ell+1} \leq n-1$.

In the rest of this section, we assume that there exists a nonconstant holomorphic mapping $f: \boldsymbol{C} \rightarrow \boldsymbol{P}^{n}(\boldsymbol{C}) \backslash S(V)$. By the same way as in the proof of Lemma 2.3, we have the following lemma:

LEMMA 4.3. (i) $k_{0}+k_{1} \leq n-1$.

(ii) If $\ell \geq 2$, then $k_{0}+k_{1} \leq n-2$.

(iii) $k_{0}+k_{\ell+1} \leq n-1$.

(iv) If $\ell \geq 1$, then $k_{0}+k_{\ell+1} \leq n-2$.

By the same argument as in the proof of Lemma 2.4, we also get the following lemma:

LEMMA 4.4. $k_{0}+\sharp\left\{j ; 2 \leq j \leq \ell\right.$ and $\left.k_{J}=1\right\} \leq n-2$.

Furthermore, we can show the following lemma by the same method as in the proof of Lemma 2.5: 
LEMMA 4.5. (i) $k_{0}+k_{1}+\sharp\left\{j ; 2 \leq j \leq \ell\right.$ and $\left.k_{J}=1\right\} \leq n-1$.

(ii) $k_{0}+k_{\ell+1}+\sharp\left\{j ; 1 \leq j \leq \ell\right.$ and $\left.k_{J}=1\right\} \leq n-1$.

\section{§5. Hyperbolicity of complements of hypersurfaces}

In this section, we prove that $\boldsymbol{P}^{n}(C) \backslash S(V)$ is complete hyperbolic and hyperbolically imbedded in $\boldsymbol{P}^{n}(\boldsymbol{C})$ in the case where $n=2,3$ and 4 . We first recall the following criterion for hyperbolicity of complements of hypersurfaces (cf. [L, Theorem 3.3]):

Lemma 5.1. Let $S$ be a hyperbolic hypersurface in $\boldsymbol{P}^{n}(\boldsymbol{C})$. Then $\boldsymbol{P}^{n}(\boldsymbol{C}) \backslash S$ is complete hyperbolic and hyperbolically imbedded in $\boldsymbol{P}^{n}(\boldsymbol{C})$ if and only if there exists no nonconstant holomorphic mapping $f: \boldsymbol{C} \rightarrow \boldsymbol{P}^{n}(\boldsymbol{C}) \backslash S$.

THEOREM 5.2. If $n=2$ and $q \geq 4$, then $\boldsymbol{P}^{2}(\boldsymbol{C}) \backslash S(V)$ is complete hyperbolic and hyperbolically imbedded in $\boldsymbol{P}^{2}(\boldsymbol{C})$.

Proof. By Theorem 3.1, $S(V)$ is hyperbolic. Suppose that there exists a nonconstant holomorphic mapping $f: \boldsymbol{C} \rightarrow \boldsymbol{P}^{2}(\boldsymbol{C}) \backslash S(V)$. If $\ell=0$, then $k_{0}+$ $k_{\ell+1} \leq 1$ by Lemma 4.3. Since $q \geq 4$, this is absurd. Hence $\ell \geq 1$. By Lemma 4.3 , we see $k_{0}+k_{1} \leq 1$. We also have $k_{\ell+1}=0$ by Lemma 4.3. Thus we conclude that the only possible type of decomposition of the second kind is of type $(0 ; 2,2, \ldots, 2 ; 1)$. This contradicts Lemma 4.5 .

Q.E.D.

THEOREM 5.3. If $n=3$ and $q \geq 6$, then $\boldsymbol{P}^{3}(\boldsymbol{C}) \backslash S(V)$ is complete hyperbolic and hyperbolically imbedded in $\boldsymbol{P}^{3}(\boldsymbol{C})$.

Proof. By Theorem 3.2, $S(V)$ is hyperbolic. Suppose that there exists a nonconstant holomorphic mapping $f: \boldsymbol{C} \rightarrow \boldsymbol{P}^{3}(\boldsymbol{C}) \backslash S(V)$. As in the proof of Theorem 5.2, we have $\ell \geq 1$. If $\ell=1$, then $k_{0}+k_{1} \leq 2$ by Lemma 4.3. In this case, it is clear that

$$
k_{0}+\left(k_{1}+1\right)+\left(k_{2}+1\right)=q \geq 6 .
$$

Thus we see $k_{2} \geq 2$, which contradicts Lemma 4.3. If $\ell \geq 2$, then $k_{0}+k_{1} \leq 1$ by Lemma 4.3 , (ii). This gives that $k_{0}=0$ and $k_{1}=1$. Thus the only possible type of decomposition of the second kind is of $\left(0 ; 2,2, \ldots, 2 ; k_{\ell+1}+1\right)$. Hence $2 \ell+k_{\ell+1}+1=q \geq 6$. On the other hand, by Lemma 4.5 , we get $k_{\ell+1}+\ell \leq 2$, and hence $k_{\ell+1} \leq-1$. This is absurd.

Q.E.D.

The least degree of the hypersurfaces in Theorem 5.3 is 31 .

We next consider the case where $n=4$ and $q=9$. We first notice the following: Suppose that there exists a decomposition of the first kind is of $(0 ; 3,3,3)$ of $V$ by $f$. Then there exist nonzero polynomials $F_{1}, \ldots, F_{s}$ of the determinants of column vectors such that $F_{j}$ are independent of $f$ and 
$F_{j}\left(v_{1}, \ldots, v_{9}\right)=0$ for some $j$, where $V=\left\{v_{1}, \ldots, v_{9}\right\}$. Indeed, by Lemma 2.2, we have

$$
\boldsymbol{v}_{2}^{(j)}-\omega_{2}^{(j)} \boldsymbol{v}_{0}^{(j)}=a_{j 0}\left(\boldsymbol{v}_{1}^{(j)}-\omega_{1}^{(j)} \boldsymbol{v}_{0}^{(j)}\right)+a_{j 1}\left(\boldsymbol{v}_{1}^{(1)}-\omega_{1}^{(1)} \boldsymbol{v}_{0}^{(1)}\right)+a_{j 2}\left(\boldsymbol{v}_{2}^{(1)}-\omega_{2}^{(1)} \boldsymbol{v}_{0}^{(1)}\right)
$$

for $j=2,3$. By applying Cramer's formula to

$$
\boldsymbol{v}_{2}^{(j)}=-\left(a_{j 1} \omega_{1}^{(1)}+a_{j 2} \omega_{2}^{(1)}\right) \boldsymbol{v}_{0}^{(1)}+a_{j 1} \boldsymbol{v}_{1}^{(1)}+a_{j 2} \boldsymbol{v}_{2}^{(1)}+\left(\omega_{2}^{(j)}-a_{j 0} \omega_{1}^{(j)}\right) \boldsymbol{v}_{0}^{(j)}+a_{j 0} \boldsymbol{v}_{1}^{(j)},
$$

we have

$$
a_{j 1}=\frac{\operatorname{det}\left(\boldsymbol{v}_{0}^{(1)}, \boldsymbol{v}_{2}^{(j)}, \boldsymbol{v}_{2}^{(1)}, \boldsymbol{v}_{0}^{(j)}, \boldsymbol{v}_{1}^{(j)}\right)}{\operatorname{det}\left(\boldsymbol{v}_{0}^{(1)}, \boldsymbol{v}_{1}^{(1)}, \boldsymbol{v}_{2}^{(1)}, \boldsymbol{v}_{0}^{(j)}, \boldsymbol{v}_{1}^{(j)}\right)}, \quad a_{j 2}=\frac{\operatorname{det}\left(\boldsymbol{v}_{0}^{(1)}, \boldsymbol{v}_{1}^{(1)}, \boldsymbol{v}_{2}^{(j)}, \boldsymbol{v}_{0}^{(j)}, \boldsymbol{v}_{1}^{(j)}\right)}{\operatorname{det}\left(\boldsymbol{v}_{0}^{(1)}, \boldsymbol{v}_{1}^{(1)}, \boldsymbol{v}_{2}^{(1)}, \boldsymbol{v}_{0}^{(j)}, \boldsymbol{v}_{1}^{(j)}\right)}
$$

and

$$
-\left(a_{j 1} \omega_{1}^{(1)}+a_{j 2} \omega_{2}^{(1)}\right)=\frac{\operatorname{det}\left(\boldsymbol{v}_{2}^{(j)}, \boldsymbol{v}_{1}^{(1)}, \boldsymbol{v}_{2}^{(1)}, \boldsymbol{v}_{0}^{(j)}, \boldsymbol{v}_{1}^{(j)}\right)}{\operatorname{det}\left(\boldsymbol{v}_{0}^{(1)}, \boldsymbol{v}_{1}^{(1)}, \boldsymbol{v}_{2}^{(1)}, \boldsymbol{v}_{0}^{(j)}, \boldsymbol{v}_{1}^{(j)}\right)} .
$$

Hence, $\omega_{1}^{(1)}$ and $\omega_{2}^{(1)}$ can be written as rational functions of the above determinants. Then, we have by (2.2)

$$
\begin{aligned}
\{\operatorname{det} & \left(\boldsymbol{v}_{0}^{(1)}, \boldsymbol{v}_{1}^{(1)}, \boldsymbol{v}_{2}^{(2)}, \boldsymbol{v}_{0}^{(2)}, \boldsymbol{v}_{1}^{(2)}\right) \operatorname{det}\left(\boldsymbol{v}_{2}^{(3)}, \boldsymbol{v}_{1}^{(1)}, \boldsymbol{v}_{2}^{(1)}, \boldsymbol{v}_{0}^{(3)}, \boldsymbol{v}_{1}^{(1)}\right) \\
& \left.\quad-\operatorname{det}\left(\boldsymbol{v}_{0}^{(1)}, \boldsymbol{v}_{1}^{(1)}, \boldsymbol{v}_{2}^{(3)}, \boldsymbol{v}_{0}^{(3)}, \boldsymbol{v}_{1}^{(3)}\right) \operatorname{det}\left(\boldsymbol{v}_{2}^{(2)}, \boldsymbol{v}_{1}^{(1)}, \boldsymbol{v}_{2}^{(1)}, \boldsymbol{v}_{0}^{(2)}, \boldsymbol{v}_{1}^{(2)}\right)\right\}^{d} \\
+ & \left\{\operatorname{det}\left(\boldsymbol{v}_{0}^{(1)}, \boldsymbol{v}_{2}^{(3)}, \boldsymbol{v}_{2}^{(1)}, \boldsymbol{v}_{0}^{(3)}, \boldsymbol{v}_{1}^{(3)}\right) \operatorname{det}\left(\boldsymbol{v}_{2}^{(2)}, \boldsymbol{v}_{1}^{(1)}, \boldsymbol{v}_{2}^{(1)}, \boldsymbol{v}_{0}^{(2)}, \boldsymbol{v}_{1}^{(2)}\right)\right. \\
& \left.\quad-\operatorname{det}\left(\boldsymbol{v}_{0}^{(1)}, \boldsymbol{v}_{2}^{(2)}, \boldsymbol{v}_{2}^{(1)}, \boldsymbol{v}_{0}^{(2)}, \boldsymbol{v}_{1}^{(2)}\right) \operatorname{det}\left(\boldsymbol{v}_{2}^{(3)}, \boldsymbol{v}_{1}^{(1)}, \boldsymbol{v}_{2}^{(1)}, \boldsymbol{v}_{0}^{(3)}, \boldsymbol{v}_{1}^{(3)}\right)\right\}^{d} \\
+ & \left\{\operatorname{det}\left(\boldsymbol{v}_{0}^{(1)}, \boldsymbol{v}_{2}^{(2)}, \boldsymbol{v}_{2}^{(1)}, \boldsymbol{v}_{0}^{(2)}, \boldsymbol{v}_{1}^{(2)}\right) \operatorname{det}\left(\boldsymbol{v}_{0}^{(1)}, \boldsymbol{v}_{1}^{(1)}, \boldsymbol{v}_{2}^{(3)}, \boldsymbol{v}_{0}^{(3)}, \boldsymbol{v}_{1}^{(3)}\right)\right. \\
& \left.-\operatorname{det}\left(\boldsymbol{v}_{0}^{(1)}, \boldsymbol{v}_{1}^{(1)}, \boldsymbol{v}_{2}^{(2)}, \boldsymbol{v}_{0}^{(2)}, \boldsymbol{v}_{1}^{(2)}\right) \operatorname{det}\left(\boldsymbol{v}_{0}^{(1)}, \boldsymbol{v}_{2}^{(3)}, \boldsymbol{v}_{2}^{(1)}, \boldsymbol{v}_{0}^{(3)}, \boldsymbol{v}_{1}^{(3)}\right)\right\}^{d} \\
= & 0 .
\end{aligned}
$$

Hence, by permutations, we get polynomials $F_{j}$ with the above property, and the number of polynomials $s$ is 9 !. Also, if there exists a decomposition of the second kind of $(0 ; 3,3 ; 3)$ of $V$ by $f$, then $F_{j}\left(v_{1}, \ldots, v_{9}\right)=0$ for some $j$. By the same way, if there exists a decomposition of the second kind of $(0 ; 3,3,2 ; 1)$ of $V$ by $f$, then we have nonzero polynomials $G_{1}, \ldots, G_{t}$ such that $G_{k}$ are independent of $f$ and $G_{k}\left(v_{1}, \ldots, v_{9}\right)=0$ for some $k$. Indeed, by Lemma 4.2 , we have

$$
\boldsymbol{v}_{j}^{(2)}-\omega_{j}^{(2)} \boldsymbol{v}_{0}^{(2)}=a_{j 1}\left(\boldsymbol{v}_{1}^{(1)}-\omega_{1}^{(1)} \boldsymbol{v}_{0}^{(1)}\right)+a_{j 2}\left(\boldsymbol{v}_{2}^{(1)}-\omega_{2}^{(1)} \boldsymbol{v}_{0}^{(1)}\right)+a_{j 3}\left(\boldsymbol{v}_{1}^{(3)}-\omega_{1}^{(3)} \boldsymbol{v}_{0}^{(3)}\right)
$$

for $j=1,2$. By Cramer's formula, we get

$$
\omega_{j}^{(2)}=\frac{\operatorname{det}\left(\boldsymbol{v}_{j}^{(2)}, \boldsymbol{v}_{0}^{(1)}, \boldsymbol{v}_{1}^{(1)}, \boldsymbol{v}_{2}^{(1)}, \boldsymbol{v}_{1}^{(3)}-\omega_{1}^{(3)} \boldsymbol{v}_{0}^{(3)}\right)}{\operatorname{det}\left(\boldsymbol{v}_{0}^{(2)}, \boldsymbol{v}_{0}^{(1)}, \boldsymbol{v}_{1}^{(1)}, \boldsymbol{v}_{2}^{(1)}, \boldsymbol{v}_{1}^{(3)}-\omega_{1}^{(3)} \boldsymbol{v}_{0}^{(3)}\right)}
$$


Note that $\omega_{1}^{(3)}$ is a $d$-th root of -1 . By (2.2) we get polynomials $G_{k}$, and the number of polynomials $t$ is $9 ! \times d$. Let $\mathscr{V}$ be a proper algebraic subset of $C^{45}$ defind by

$$
\mathscr{V}=\left(\bigcup_{J=1}^{s}\left\{F_{j}\left(\boldsymbol{v}_{1}, \ldots, \boldsymbol{v}_{9}\right)=0\right\}\right) \cup\left(\bigcup_{J=1}^{t}\left\{G_{j}\left(\boldsymbol{v}_{1}, \ldots, \boldsymbol{v}_{9}\right)=0\right\}\right) .
$$

THEOREM 5.4. If $q=9$ and $V \notin \mathscr{V}$, then $\boldsymbol{P}^{4}(C) \backslash S(V)$ is complete hyperbolic and hyperbolically imbedded in $\boldsymbol{P}^{4}(\boldsymbol{C})$.

Proof. Suppose that there exists a nonconstant holomorphic mapping $f: C \rightarrow S(V)$. Then by Lemmas 2.3 and 2.5 , it is easy that the only possible type of decomposition of the first kind by $f$ is of type $(0 ; 3,3,3)$. If $\boldsymbol{P}^{4}(\boldsymbol{C}) \backslash S(V)$ is not Brody hyperbolic, then we also see that the only possible types of decomposition of the second kind are of types $(0 ; 3,3 ; 3)$ and $(0 ; 3,3,2 ; 1)$ by Lemmas 4.3 and 4.4. Hence $V \notin \mathscr{V}$ yields our assertion.

Q.E.D.

By Theorem 5.4, we obtain hyperbolic hypersufaces of degree $d \geq 73$ in $\boldsymbol{P}^{4}(\boldsymbol{C})$ whose complements are complete hyperbolic and hyperbolically imbedded in $\boldsymbol{P}^{4}(\boldsymbol{C})$.

Example 5.5. Let $\boldsymbol{v}_{1}={ }^{t}(1,0,0), \boldsymbol{v}_{2}={ }^{t}(0,1,0), \boldsymbol{v}_{3}={ }^{t}(0,0,1), \boldsymbol{v}_{4}={ }^{t}(a, b, c)$ and $V=\left\{\boldsymbol{v}_{1}, \boldsymbol{v}_{2}, \boldsymbol{v}_{3}, \boldsymbol{v}_{4}\right\}$. The condition (A1) is equivalent to $a b c \neq 0$, (A2) to $a^{d} \neq-1, b^{d} \neq-1, c^{d} \neq-1$, and (A3) to $a^{d}+(-b)^{d} \neq 0, b^{d}+(-c)^{d} \neq 0$, $c^{d}+(-a)^{d} \neq 0$. Hence, if $a, b, c$ satisfy the above conditions and $d \geq 13$, then the hypersurface in $\boldsymbol{P}^{2}(\boldsymbol{C})$ defined by

$$
z_{0}^{d}+z_{1}^{d}+z_{2}^{d}+\left(a z_{0}+b z_{1}+c z_{2}\right)^{d}=0
$$

is hyperbolic and its complement is complete hyperbolic and hyperbolically imbedded in $\boldsymbol{P}^{2}(\boldsymbol{C})$. Suppose that this hypersurface has a singular point $\left(p_{0}: p_{1}: p_{2}\right)$. Then $p_{0}^{d-1}+a\left(a p_{0}+b p_{1}+c p_{2}\right)^{d-1}=0, \quad p_{1}^{d-1}+b\left(a p_{0}+b p_{1}+\right.$ $\left.c p_{2}\right)^{d-1}=0$ and $p_{2}^{d-1}+c\left(a p_{0}+b p_{1}+c p_{2}\right)^{d-1}=0$. It is trivial that $P:=a p_{0}+$ $b p_{1}+c p_{2} \neq 0$. Hence we have $p_{0}=\omega P, p_{1}=\eta P$ and $p_{2}=\xi P$ for some $(d-1)$-th roots $\omega, \eta, \xi$ of $-a,-b,-c$, respectively. Hence we get $\omega a+\eta b+$ $\xi_{c}=1$. Therefore, this hypersurface is smooth if $\omega a+\eta b+\xi c \neq 1$ for any $(d-1)$-th roots $\omega, \eta, \xi$ of $-a,-b,-c$, respectively. For example, if $a=1 / 2$, $b=1 / 4, c=1 / 8$, these four conditions are satisfied.

M. B. Zaindenberg showed in $[Z]$ that the existence of a smooth hyperbolic hypersurface of degree 5 in $\boldsymbol{P}^{2}(\boldsymbol{C})$ whose complement is complete hyperbolic and hyperbolically imbedded in $\boldsymbol{P}^{2}(\boldsymbol{C})$. On the other hand, K. Azukawa and $\mathbf{M}$. Suzuki $[\mathrm{AS}]$ gave an explicit equation defining such a hypersurface of degree 14. The degree 13 of our example is lower than it. 


\section{REFERENCES}

[AS] K. AzuKawa AND M. SuzUKI, Some examples of algebraic degeneracy and hyperbolic manifolds, Rocky Mountain J. Math., 10 (1980), 655-659.

[B] R. BRody, Compact manifolds and hyperbolicity, Trans. Amer. Math. Soc., 235 (1978), 213-219.

[BG] R. BRody AND M. Green, A family of smooth hyperbolic hypersurfaces in $\boldsymbol{P}_{3}$, Duke Math. J., 44 (1975), 873-874.

[D] J.-P. Demailly, Algebraic criteria for Kobayashı hyperbolic projective varieties and jet differentials, Algebraic Geometry held at Santa Cruz, July 1995 (J. Kollár et al. eds.), Proc. Sympos. Pure Math., 62-2, Amer. Math. Soc., Providence, 1997, 285-360.

[F] H. Fujimoto, On meromorphic maps into the complex projective space, J. Math. Soc. Japan, 26 (1974), 272-288.

[G] M. L. GreEN, Some Picard theorems for holomorphic maps to algebraic varieties, Amer. J. Math., 97 (1975), 43-75.

[K] H. H. KhoAI, Hyperbolic surfaces in $\boldsymbol{P}^{3}(\boldsymbol{C})$, Proc. Amer. Math. Soc., 125 (1997), 35273532.

[L] S. LANG, Introduction to Complex Hyperbolic Spaces, Springer-Verlag, New York, 1987

[MN] K. MASUdA AND J. NoguCHI, A construction of hyperbolic hypersurface of $\boldsymbol{P}^{n}(\boldsymbol{C})$, Math. Ann., 304 (1996), 339-362.

[N] A. NADEL, Hyperbolic surfaces in $\boldsymbol{P}^{3}$, Duke Math. J., 58 (1989), 749-771.

[S] M. ShIRosaki, On some hypersurfaces and holomorphic mappıngs, Koda1 Math. J., 21 (1998), 29-34.

[Z] M. G. ZAIDENBERG, Stability of hyperbolic imbeddedness and constraction of examples, Math. USSR-Sb., 63 (1989), 351-361.

Department of Mathematical Sciences

College of ENGINEERING

Osaka Prefecture UnIVERSity

SAKAI, 599-8531, JAPAN 